\title{
COVID-19 Pandemic: A Qualitative Investigation into the Experiences of COVID Infected Sample of Nepalese People
}

\begin{abstract}
Harihar Ghimire (PhD Scholar)*
"Physical fitness is not one of the most important keys to a healthy body; it is the basis of dynamic and creative intellectual activity." John F. Kennedy
\end{abstract}

\begin{abstract}
The main aim of this study is to explore COVID-19 and its experiences of Nepalese people. Primary data were collected through telephone interviews and secondary data were used from different published and unpublished sources in this study. This study is basically based on qualitative data. Total 30 respondents were selected through snowball sampling methods. Among the main three findings, the first is the sources of COVID-19 may or may not be identify and syndromes may or may not be seen in every individual during first and second wave of COVID-19.The another conclusion is that the experiences were vary from individual to individual and were felt almost same experiences. However, the duration of recovery days may be different. Lastly, almost all respondents had some kind of experience in post COVID-19 syndromes. But the post COVID-19 side effects have been decreasing slowly. This paper covers only thirty affected respondents' experiences of residents of Kathmandu. The value of the paper is to inform people about the nature of COVID-19 in various stages.
\end{abstract}

Key words: COVID-19, COVID symptoms, syndromes \& experiences.

\section{Introduction}

Society and infectious diseases have had a profound and lasting effect on societies throughout history. All the diseases don't spread worldwide. However, few diseases have been spreading worldwide, which is called pandemic. There are numerous lists of Pandemic in human

${ }^{*}$ Mr. Ghimire is a Lecturer at the Department of Sociology, Patan Multiple Campus, TU, Lalitpur, Nepal.Email: ghimireharihar10@gmail.com 
history. Some examples are Spanish flu (1918-1920), HIV/AIDS pandemic (1981-present), Influenza pandemic (1957-1958), and Hong Kong flu (1968-1969) etc. If we look at more recent human history, there have been different viruses like SARS in 2003, influenza H1N1 in 2009, MERS in 2012, Ebola in 2014, Zika Virus in 2016 have been reported. Currently, the COVID-19 pandemic is spreading in every individual in the globe.

Around December 2019, an increasing number of pneumonia cases of unknown origin were reported in the Chinese city of Wuhan (Chen et al, 2020). This increasing number of pneumonia cases in China-scientists later believe this to be caused by a virus-which was named by World Health Organization (WHO) as 2019-nCov with associated illness of COVID-19.

Various scholars have been defining the COVID-19 pandemic from the beginning. A novel coronavirus or coronavirus 2 (SARS-CoV-2) was first recognized in a seafood market in Wuhan City in China in the last week of December 2019. Likewise, Mohanty et al. (2020) viewed that Coronavirus disease 2019 (COVID-19) is an infectious disease caused by the newly detected severe acute respiratory syndrome coronavirus 2 (SARS-CoV-2) which primarily affects the respiratory tract and particularly the lungs. Another scholar Wu et al. (2020) explain that the contagious respiratory disease caused by this novel coronavirus is called coronavirus disease 2019 or, in short, COVID-19 Pneumonia cases. The date of symptoms present in the first patient identified in Wuhan, China, was $1^{\text {st }}$ December 2019. Likewise, Panthee et al. (2020) say that after the official notice regarding COVID-19 was received by the World Health Organization on 31 December 2019. The World Health Organization and the global community have been working continuously to restrain this disease, which quickly became a global pandemic. The novel coronavirus is one of the pandemics of human history. This virus has been globalized in a very short period.

Being a pandemic, the nature of this disease is globalizing all over the world. Nepal is also affected from the third week of January 2020.Meanwhile, Bastola et al. (2020) mention that the first and initial case was detected on 23 January 2020 in a patient who had returned from Wuhan, China. The diagnostic approaches in Nepal were not established at the same time, so the sample had to be sent overseas for testing in order to be making the first identification and diagnosis. Once the first case was detected, the government of Nepal has been working on reducing the spread and impact of the disease. As we know that health facilities like advanced public laboratories and other services are not satisfactory in Nepal. Meanwhile, Prasain (2020) mentions that the public laboratories in Nepal did not have testing agents required for testing and there were no suspected cases needing testing. Hence, the samples were sent to Hong Kong for testing, which showed positive results for COVID-19.Panthee et al. (2020) further mention that 2 months after the first case, a second case was diagnosed through domestic testing on 23 March on a returnee from France. In response to this, Nepal decided to close its international borders 
completely and imposed a national-level lockdown until 14 June 2020 for 74days. Seeing no progress, the lockdown was further extended. In addition, the government established a COVID-19 crisis management system by the recommendation of exports from various sectors and focused on setting up temporary hospitals, quarantine, isolation and intensive care unit beds in each province.

Meanwhile, Tamang et al. (2020) explain that as the Coronavirus (COVID-19) outbreak continues to evolve, countries around the world have introduced preventive measures to reduce human-to-human transmission in areas where the virus that causes COVID-19 is already circulating in the population. Public health measures include quarantine, which involves the restriction of the movement of sick people from the rest of the population. With the rapid increase in the number of COVID-19 positive cases, the Government of Nepal has also introduced quarantine for the people having COVID-19 positive cases in different schools, hotels which include the citizens and also returning migrant workers and their accompanying family members.

Now, we can say that COVID-19 as a pandemic-mostly affects every individual of the world. Nepal is also affected by this disease from the very beginning. However, the second wave of pandemic is more dangerous and harmful in Nepal. For the same, the first wave started with a few syndromes which were easier to identify and care about the COVID-19 cases. However, the second wave of this disease is not compulsory to see the syndromes.

As the researcher writes the memoir, almost one and half year later today (as of $6 \mathrm{pm}, 4^{\text {th }}$ June 2021) a total of 171708011 COVID-19 cases worldwide have been confirmed, out of whom 3697151 have died. In a short period of time, COVID-19 pandemic became globalized which affected almost all countries of the world. For the same, today (as of 8 am 12 $2^{\text {th }}$ une 2021) a total of 604396 COVID-19 cases in Nepal have been confirmed, out of whom 8,305 have died. The world is affected by second wave of COVID-19 which is very quick transformative character compare to first wave of COVID-19. As we know that WHO, health experts and other health practitioners frequently said that no syndrome could be or could not seen in every COVID-19 cases in second wave worldwide. However, in the first wave of COVID-19 cases few syndromes could be seen in almost every case. This study focuses on both the cases of first and second wave of COVID-19 in Nepal.

During the globalization of COVID-19 pandemic, different symptoms have been noticed in different affected individuals worldwide. This virus spread to all continents (except Antarctica). It affects every individual in the world and has been affecting now. There are three stages of this disease: pre-COVID-19 syndromes, COVID-19 incubation Period and post-COVID-19 syndromes. This study covers first and second wave of COVID-19 affected people of Kathmandu. Generally, the syndromes of COVID-19 are fever, cough, difficulty breathing, tiredness, muscle aches, headaches, loss of taste and smell, sore 
throat, runny nose, vomiting Diarrhea etc. Likewise, COVID-19 incubation period is a very difficult period for an infected person. In simple sentences, an incubation period of a disease describes the duration between initial exposure and onset of disease syndromes. According to the World Health Organization, the estimated range of SAR-Cov-2 was 1-14 days. However, the average incubation period was estimated to be 5-6 days. One important thing to be remembered is that the incubation period may vary from person to person.

Table-1: Distribution of the highest number of COVID-19 affected by district (12 ${ }^{\text {th }}$ June)

\begin{tabular}{|c|l|c|}
\hline SN & District & Number \\
\hline 1 & Kathmandu & 198384 \\
\hline 2 & Lalitpur & 35883 \\
\hline 3 & Rupandehi & 28347 \\
\hline 4 & Morang & 27298 \\
\hline 5 & Bhaktapur & 21391 \\
\hline 6 & Kaski & 21150 \\
\hline 7 & Banke & 17493 \\
\hline 8 & Sunsari & 16101 \\
\hline 9 & Kailali & 15873 \\
\hline 10 & Jhapa & 13396 \\
\hline 11 & Dang & 12210 \\
\hline 12 & Kavrepalanchow & 10343 \\
\hline 13 & Surkhet & 10265 \\
\hline 14 & Makawanpur & 8918 \\
\hline 15 & Dhanusha & 8746 \\
\hline
\end{tabular}

Source: Ministry of Health and Population, 2021

The above table shows that Kathmandu is a highly affected district by COVID-19 pandemic. Kathmandu is the capital of Nepal and still every person has to come to Kathmandu for multiple reasons even though Nepal is a Federal State. From 77 districts everyday thousands of people entered the capital for multiple works. Naturally, the large number of people affected daily in Kathmandu by COVID-19. The second and third highly affected districts are Lalitpur and Rupandehi respectively. Among highly affected 15 districts, Dhanusha is the lowest number in affected cases. 


\section{Literature Review}

Various studies on experiences of COVID-19 affected people have been done. Malande (2020) writes that 'My story I think is similar to that of many workers affected by COVID-19. I had been significantly exposed and ran a real risk of coronavirus. About four days later, I developed a persistent headache unresponsive to Paracetamol and flu- like symptoms. I decided to get tested for coronavirus, and the result confirmed it, I was COVID-19 PCR positive' (p.1510). However, Malande (2020) describes her experiences during COVID-19 period that 'I have never experienced the kind of pain and myalgia. I suffered during illness, it was serving and debilitating. I also had extreme fatigue, and could hardly lift a twenty liter jerrican of water' ( $p$ 1511). As we know that there is few study have been done on the effects of COVID-19 pandemic. This pandemic does not see any group, class, color, age, region etc. Meanwhile, Malande (2020) further experiences that 'On two separate nights in my first 5- 10 days after COVID-19 diagnosis, I suffered from episodes of breathlessness and air hunger. Which was as if I was drowning? This puzzled me, especially knowing that I am young, fit, and have no comorbidities whatsoever,(p.1511). Different people experienced differently during COVID-19 pandemic. Meanwhile, Malande (2020) writes of her experiences that ' I also experienced weakness, tiredness and drenching night sweats, alongside the irritating persistent dry cough, headache that was almost splitting my head into two - that only made the muscle and joint pains ever worse' (p.1511).

Generally the effect of COVID-19 could be seen for a long time. However, all cases could not be generalized. We can explain the various experiences of COVID -19 worldwide. Malande(2020) mentions that 'As I slowly recovered from the hypoxic spells, I recovered for my pulse oximeter and what a relief when profoundly I was averaging 94-95\% oxygen saturation in room air. The COVID-19 infection pushes you to the limit, and at some point, you will have to summon all energy and inner strength to stay afloat' (p.1511). Studies have been shown that the COVID-19 Pandemic, consistent with previous pandemics, is negatively and disproportionately impacting on particular groups of people such as older adults, migrants' worker, women, health workers, and overseas students (Rajkumar, 2020, Gates, 2020, The World Bank, 2020)

After reviewing the available literature, few studies on experiences of COVID-19 have been done all over the world and as well as in Nepal. However, this type of study is very rare. So, the following research questions have been raised in this study:

i) What are the possible sources of transmission and symptoms before COVID-19 positive? 
ii) What experiences have had the individuals during COVID-19 positive?

iii) What are the post-COVID-19 side effects experienced by the individuals?

\section{Methods}

A numerous people have been affected in the first and second wave of COVID-19 pandemic in Nepal. In this study, the researcher interviewed thirty respondents who were selected purposely from residents of Kathmandu district. The snowball sampling method was used where respondent selects the respondents. For the same, the researcher first of all collected the phone number of COVID-19 affected people and then interviewed individually. The highest number of COVID-19 affected people is found in Kathmandu. So, Kathmandu is purposely selected. The face-to-face interview is not possible and suitable in this pandemic situation. The primary data were collected through the telephone interview method and secondary data were collected through various available published and unpublished documents in this study.

\section{Results}

Many people of the world have been affected by COVID-19. At the time of writing this paper, the first wave of this pandemic has already ended and the second wave is affecting people in Nepal as well as in the globe. The researcher interviewed with COVID-19 affected thirty people and collected information of experiences before-during-post COVID-19.

Table 2: Distribution of COVID-19 affected respondents by Sex

\begin{tabular}{|c|l|c|c|}
\hline SN & Particulars & Respondents & Percent \\
\hline 1 & Male & 23 & 77 \\
\hline 2 & Female & 7 & 23 \\
\hline Total & & $\mathbf{3 0}$ & $\mathbf{1 0 0}$ \\
\hline
\end{tabular}

Source: Field Survey, 2021

Table 2 shows that the majority of the respondents (77\%) are found male and only 23 percentages are female. The literatures also show that males affected are higher than females in Nepal as well as in the world. In my observation, male are always have to go outside for multiple purposes in comparison with females. However, females are less exposed in outside activities in this pandemic situation. My observation and also available data also shows that male is more affected than females. 
Table 3: Distribution of COVID-19 affected respondents by occupation

\begin{tabular}{|c|l|c|c|}
\hline SN & Particulars & Number & Percent \\
\hline 1 & Doctor & 10 & 33 \\
\hline 2 & Nurse & 8 & 27 \\
\hline 3 & Teacher & 6 & 20 \\
\hline 4 & Government Officials & 4 & 13 \\
\hline 5 & Police and Army & 2 & 7 \\
\hline Total & & $\mathbf{3 0}$ & $\mathbf{1 0 0}$ \\
\hline
\end{tabular}

Source: Field Survey, 2021

The above table shows that the majority of 33 percent Doctors and 27 percentage Nurses are found affected by COVID-19. It also found that 20 percentages are university teachers. The remaining respondents are government employees and security which comprises 13 percent and 7 percent respectively. The relation of COVID-19 and affected individuals are very meaningful. Doctors and Nurses are closely working with affected people. So, they are found in larger numbers than others.

Table 4: Distribution of incubation period of COVID-19 by the respondents

\begin{tabular}{|c|l|c|c|}
\hline SN & Incubation Period & Number & Percent \\
\hline 1 & $1-3$ days & 6 & 20 \\
\hline 2 & $1-6$ days & 12 & 40 \\
\hline 3 & 1-9days & 5 & 16 \\
\hline 4 & $1-14$ days & 5 & 16 \\
\hline 5 & More than 14 days & 2 & 7 \\
\hline Total & & $\mathbf{3 0}$ & $\mathbf{1 0 0}$ \\
\hline
\end{tabular}

Source: Field Survey, 2021

There are not the same experiences felt by all COVID-19 affected people in the globe. Nepal also had felt the same experiences. This study also found that there are varieties of experiences felt by respondents in the capital of Nepal during COVID-19. The majority or 40 percent of respondents felt their incubation period remained 1-6 days. They said that after the PCR test positive they felt very painful. They also added that no one should ignore this pandemic. Everyone should be alert or should not be taken as simple diseases. It is a very long term affected disease. They shared their experiences that from 1- 6 days 
they felt painful and isolated ever. Among 40 percentages, 95 percent felt well within 5-6 days. According to WHO and Centre for Disease Control and Prevention, the average incubation period was estimated to be 5-6 days. We can that the experiences felt by Nepalese are likely to available literatures,

The second highest number or 20 percent of respondents said their incubation period is very short i.e. 1-3 days. However, they felt very difficult in that short period. All 6 respondents felt easy after 3 days. For the same, 16 percent of respondents felt difficulty for up to 9 days. The same number felt difficulty from 1- 14 days. Only 7 percent of respondents felt very difficult because they were treated in the Intensive Care Unit (ICU). According to WHO, the estimated range of incubation period of SAR-Cov-2 was from 1-14 days. The result of this study also found that 93 percent of respondents recovered within 14 days.

This study is basically focused on experiences of respondents felt in before, during and post COVID-19. The following sub-topic deals with the experiences felt by affected respondents in this study.

\section{Sources of Transmission and Symptoms before COVID-19 Positive}

In my observation, the common source of transmission of COVID-19 are at the time of serving COVID-19 patients in Hospital, walking in a crowd area without wearing mask, long trip with friends, having anywhere or food, working in Public relation office, interacting with students, travelled in public transport and many people did not know the sources of transmission. In this study, the information given by respondents is not different from general knowledge. Majority of respondents did not know the actual source transmission. They said that once he had traveled with friends, some said that I walked in crowded places without a mask; some said that having food outside home may be the source of transmission of COVID-19. My own experience may be interesting. Last year in 2020, we four friends went to Ramechhap to give a courtesy visit to one of our friends whose father recently died. We went there in a private car wearing masks. However, the day after tomorrow after returning to Kathmandu, one of my friends phoned me and said that my PCR test is positive. So, you have to isolate yourself and take care. For the same, my test and smell had already lost. The remaining friend did not have any problem. It means no one of us could identify the source of Transmission of COVID-19.

After analyzing the information given by respondents, 45 percent of respondents said that closely working with COVID-19 affected people in hospitals was the source of transmission of COVID-19. Meanwhile, the 2/3 respondents said that they did not know 
the actual source of transmission. The remaining few respondents shared that while caring for the COVID-19 affected people was the source of transmission of COVID-19. So, it is not certain that everyone could know the source of transmission of COVID-19.

\section{The Experiences of COVID infected Individuals during COVID-19 Positive}

The experiences of people of COVID-19 during the affected period may be varying from individual to individual. People who are older have a higher risk of serious illness from COVID-19, and the risk increases with age. The researcher found that 40 percent of respondents recovered within 6 days. However, thirty percent of respondents recovered within fourteen days. The remaining 7 percent recovered after more than 14 days who were treated in ICU. During COVID-19 the common symptoms were seen in every respondent. Shortness of breathing or difficulty breathing, muscle aches, chills, sore throat, runny nose, headache, vomiting, diarrhea, rash etc. were experienced by almost all respondents. However, all symptoms were not experienced by everyone in the first wave of COVID-19 pandemic. In the second wave of pandemic, no symptoms could be experienced by many COVID-19 affected people. In my observation, my friend experienced a few symptoms such as muscle aches, sore throat, headache etc. during the isolation period of COVID-19. Another friend who did not do the PCR test experienced multiple symptoms such as shortness of breathing or difficulty breathing, muscle aches, sore throat, runny nose, headache, diarrhea, etc. who faced more difficulty than other two friends

After confirming the COVID-19, after discussing with me one of my friends decided to isolate me at home by saying and accepting my suggestion that home isolation is better from multiple sides than hospital. During COVID-19, we talked minimum twice a day. My focus always went to know the difficulty during COVID-19 pandemic. I never felt tasteless and smelly. However, he was suffering from fever, back pain, laziness, weakness,

\section{The Post-COVID-19 Experienced by the Individuals}

According to my friend, he felt back pain, tiredness, weakness etc. in post-COVID-19 that remained for 15 days. Now, he has no post-COVID-19 syndromes and feels easy and fully recovered. The 80 percent respondents recovered within 9 days. However, few respondents or 16 percent recovered within 14 days. The remaining was recovered after 14 days. The majority or 40 percent of respondents recovered within 6 days. It is found that the fast recovered respondents did not feel very trouble in post COVID-19.However, 80 percent of respondents felt a few syndromes such as weakness, back pain, memory loss, tiredness, and back pain etc. Remaining 20 percent felt very few syndromes like 
tiredness, back pain, and memory loss etc. It is also found that 20 percent of respondents who recovered within 3 days felt very difficulty with laziness, tiredness, and fever etc. They felt easy after 3 days. However, among them few respondents experienced memory loss, tiredness, frustrations etc. Likewise, 32 percent respondents who recovered within 9 days and 14 days experienced almost the same long term side effect of COVID-19. They shared that they did not think that this disease is very dangerous and quickly suggested that they do not overlook the COVID-19 pandemic. So, everyone must follow the minimum health protocol suggested by WHO and Ministry of Health and Population of Nepal. Lastly, only two respondents felt very difficulty in post COVID-19.They said that they are still afraid when they remember the past.

\section{Analysis and Discussion}

The majority of infected respondents are working in the front line in COVID-19 pandemic. It means those who are used to work or go outside the home have a high possibility of infection by COVID-19. The majority of respondents recovered within 5-6 days. However, some respondents recovered within 14 days. And some respondents felt easy within three days. It means the recovery days are varying to every infected individual. Likewise, almost all respondents did not know the actual source of transmission. Only some respondents know the source of transmission. During COVID-19 positive period, the common symptoms such as shortness of breathing or difficulty breathing, muscle aches, chills, sore throat, runny nose, headache, vomiting, diarrhea, rash etc. are seen in every respondent. However, all symptoms were not experienced by everyone but few symptoms were experienced by every individual.

Almost all respondents felt some syndromes such as weakness, back pain, memory loss, tiredness, and back pain etc in post-COVID. The respondents who recovered within 3 days did not fell any difficulty in post COVID-19. However, few respondents experienced such as memory loss, tiredness, frustrations, and back pain etc. since multiple months.

\section{Concluding Remarks}

With exceptional cases almost all the people of the world have been affected by COVID-19 pandemic. Present study focuses only on the experiences of thirty professionals in Kathmandu Valley and was selected through snowball sampling. The first conclusion of this study is that the sources of COVID-19 may or may not identify and syndromes may or may not be seen in every individual during the first and second wave of COVID-19. The another conclusion is that the experiences vary from individual to individual and all were felt almost same experiences. However, the duration of recovery days may be 
different. Lastly, almost all respondents had some kind of experience in post COVID-19 syndromes even they did not feel very difficulty.

\section{References}

Bastola, A., Sah, R., Morales, A. J. R., Lal, B. K., Jha, R., \& OJha, C. (2020). The first 2019 coronavirus case in Nepal. The Lancet, infectious Disease, 20(3), March 2020.

Chen, Q., Mining, L., Yamin, L., Jincal, G., Dongxue, F., Ling, W., Li, H., Caihus, S., Yiwen, C., \& Xiaojuan, L.(2020). Mental health care for medical staff in China during the COVID-19 outbreak. Lancet Psychiatry. https://doi.org/10.1016/s22150366(20)300780x.

Gates, M. (2020). The pandemic's toll on women: COVID-19 is gender-blind, but not gender-neutral. Foreign Affairs. https://www.foreignaffairs.com/articles/ world/2020-07-15/melinda-gates-pandemic-toll-women

World Bank Group (WBG). (2020). Gender dimensions of the COVID-19 pandemic. Policy note. The World Bank Group. https://openknwledge.woldbank.org/ bitstream/handle/10986/33622/Gender-Dimensions-of-\%20the-COVID-19Pandemic.pdf?sequence $=1$

Malande, O. O. (2020). My COVID-19 experiences: Picking up the pieces. African Health Science 20(4). https://dx.doi.org./10.4314/ahs.v20i4.4

Ministry of Health and Population (MoHP). (2020). COVID-19 dashboard. Government of Nepal. Kathmandu, Nepal. https://covid19.mohp.gov.np/

Mohanty, S. K., Satapathy, A., Naidu, M. M., Mukhopadhya, S., Sharma, Borton, L. M., \& Parwani, A. V. (2020). Severe acute respiratory syndrome coronavirus-2 (SARSCOV-2) and coronavirus disease 19 (COVID-19)-anatomic pathology perspective on current knowledge. Diagnostic Pathology, 15(1). Doi:.1186/s13000-020-01017-8

Panthee, B., Dhungana, P., Poudel, N. A., Gyawali, S., \& Panthee, S., (2020). COVID-19: The current situation in Nepal. New Microbes and New Infection, 37.

Rajkumar, R. P. (2020). COVID-19 and mental health: A review of the existing literature. Elsevier Health Emergency Collection. https:/www.ncbi.nlm.nih.gov/pmc/ articles /PMC 7151415

Tamang, P., Mahato, P., Shahi, P., \& Simkhada, P.(2020). COVID-19 Quarantine; A key part of prevention in Nepal. DOI:10.3126/jkahs.v3i0.29432 